\title{
Índice Valvar \& Escore Valvar. Uma nova forma de Comunicação do continuum da História Natural da Valvopatia
}

\author{
Max Grinberg e Guilherme Sobreira Spina \\ São Paulo, SP
}

Informações da anamnese, exame físico e exames complementares qualificam e quantificam o continuum patológico desencadeado pela lesão valvar. Acompanhar um paciente com cardiopatia valvar, que costuma ter história natural longa, é construir um pari-passu de comparações. Três tipos de comportamentos fazem parte da nossa prática do dia-a-dia: 1) observação da adaptação cardiovascular, incluindo prevenção do fator etiopatogênico valvar (febre reumática ou endocardite infecciosa); 2 ) intervenção sobre influências modificadoras da história natural (surto reumático, endocardite infecciosa, gestação); 3) substituição da história natural por história pós-operatória.

Cada momento da avaliação valvar representa um elo entre passos precedentes e previsões sobre comportamentos seguintes. Há o momento em que o caso é tão somente um "sopro", e que pressupõe muito tempo de convivência recomendada com a história natural; há o momento da lesão valvar grave com o paciente permanecendo com boa qualidade de vida; há o momento "de fato doente" assim visto devido à manifestação de sintomas; há o momento que ultrapassou o ponto da história natural em que 0 cardiologista se obrigaria a recomendar intervenção sobre a essência anatômica da cardiopatia ${ }^{1,2}$.

Sob a óptica da praticabilidade, acompanhar um portador de valvopatia é acumular respostas a quatro quesitos, cada um simbolizado por uma letra: 1) qual a gravidade anatomoclínica da lesão (V); 2 ) qual a repercussão sobre a função miocárdica (M); 3) qual o grau de eventual obstrução em artéria coronária (C) 4) qual o efeito sobre os níveis de pressão na artéria pulmonar $(P)$ ?

Entendemos que reunir quatro respostas sob a sigla VMCP contribui para a caracterização do momento clínico e a comunica- ção interconsulta. Estamos propondo uma classificação $4 \times 4$, ou seja, ela inclui 4 variáveis, cada uma delas com 4 categorias. 0 VMCP representa índice valvar (ex: $\left.\mathrm{V}_{3} \mathrm{M}_{2} \mathrm{C}_{1} \mathrm{P}_{2}\right)$ e fundamenta 0 escore valvar ( $3+2+1+2=8$ no caso citado) quadro I.

Nossa intenção foi definir a subdivisão em categorias com base em pontos de referência habitualmente empregados. Assim, a passagem de V2 para V3 inclui o caso na classe I de recomendação para tratamento cirúrgico conforme diretriz ${ }^{1}$. A identificação do caso como M3 ou M4 implica em influência sobre o prognóstico cirúrgico.

O escore VMCP foi aplicado a 608 valvopatas submetidos a tratamento cirúrgico entre janeiro/2002 a março/2003 em nossa instituição. A média etária foi de $48.9 \pm 17$ anos, 55,3\% sexo feminino, 58,7\% de etiologia reumática.

0 grupo com VMCP > 8 teve maior tempo de internação que o grupo com escore menor ou igual a este valor $(26,6 \pm 23,1$ vs $20,9 \pm 18,7$ dias, $p=0,006)$ e maior tempo de internação em UTI pós operatória $(8,8 \pm 19,4$ vs $5,25 \pm 8,97$ dias, $p=0,029)$.

Para a mortalidade, a análise univariada demonstrou idade $(p=0,005$, odds ratio $(O R)=1,03)$, diabetes $(p=0,001, O R=1,7)$, história de insuficiência renal ( $p=0,0001,0 R=9,1)$, hospitalização prévia por insuficiência cardíaca (ICC) $(p=0,001, O R=8,9)$, reoperação $(p=0,0009, O R=3,22)$ e escore VMCP $>8(p=0,001$, $\mathrm{OR}=3,0)$. Após análise multivariada, o VMCP continuava associado à mortalidade $(p=0,034, O R=1,33)$ após ajuste para idade, reoperação, diabetes, insuficiência renal e hospitalização por ICC.

Quanto ao tempo de internação, a análise univariada demonstrou que tempo de internação maior que 10 dias estava associado

\begin{tabular}{|c|c|c|c|}
\hline \multicolumn{4}{|c|}{ Quadro I - Sistematização do Índice Valvar VMCP } \\
\hline V - Valva ou prótese & M - Miocárdio & C - Artéria coronária & $\begin{array}{l}\text { P - Pressão sistólica de artéria } \\
\text { pulmonar (pela ecocardiografia) }\end{array}$ \\
\hline V1 - Lesão valvar discreta / moderada & M1 - Fração de ejeção > 60\% & $\begin{array}{l}\text { C1 - Artérias coronárias normais ou } \\
\text { sem informação }\end{array}$ & $\mathrm{Pl}-\mathrm{PAP}<30 \mathrm{mmHg}$ \\
\hline V2 - Lesão valvar grave assintomática & $\begin{array}{l}\text { M2 - Fração de ejeção entre } \\
60 \% \text { e } 50 \%\end{array}$ & C2 - Obstrução coronárias até $60 \%$ & P2 - PAP entre 30 e $60 \mathrm{mmHg}$ \\
\hline V3 - Lesão univalvar sintomática & $\begin{array}{l}\text { M3 - Fração de ejeção entre } \\
50 \% \text { e } 30 \%\end{array}$ & $\begin{array}{l}\text { C3 - Obstrução coronária crítica } \\
\text { uniarterial }\end{array}$ & $\begin{array}{l}\text { P3 - PAP entre } 60 \text { e } \\
100 \mathrm{mmHg}\end{array}$ \\
\hline V4 - Lesão sintomática multivalvar & M4 - Fração de ejeção < 30\% & $\begin{array}{l}\text { C4 - Obstruções coronárias críticas } \\
\text { multiarteriais }\end{array}$ & P4 - PAP > 100mmHg \\
\hline
\end{tabular}

Instituto do Coração do Hospital das Clínicas da FMUSP

Endereço para correspondência - Dr. Max Grinberg - Av. Dr. Enéas de Carvalho Aguiar, 44 - Cep 05403-000 - São Paulo - SP

E-mail: max@cardiol.br e grinberg@incor.usp.br

Recebido em 28/11/2004 - Aceito em 12/01/2005 
com tabagismo $(p=0,03, O R=1,8)$, presença de fibrilação atrial $(p=0,003, O R=2,1)$ e índice VMCP $>8(p=0,01, O R=1,7)$. Após análise multivariada, o índice VMCP maior que 8 ainda estava associado a tempo de internação maior que 10 dias $(p=0,02, O R=1,3)$, após ajuste para tabagismo e presença de fibrilação atrial.
Ao adotarmos um valor de 8 como linha de corte para 0 escore valvar, obtivemos $84 \%$ de especificidade, com valor preditivo negativo de $81 \%$ para período de internação mais prolongado que 10 dias. Os pacientes com escore valvar maior do que 8 associaram-se a maior mortalidade cirúrgica imediata $(p=0,006)$.

\section{Referências}

1. American College of Cardiology/American Heart Association. Guidelines for the management of patients with valvular heart disease. Circulation 1998;98:1949-84
2. Edwards $F H$, Peterson ED, Coombs LP et al. Prediction of operative mortality after valve replacement surgery. J Am Coll Cardiol 2001; 37:691-974. 